\title{
Rationierung oder Rationalisierung?
}

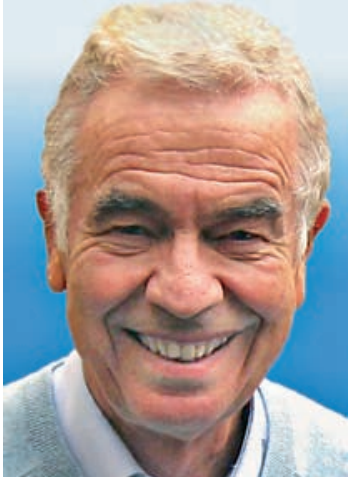

Hans Stalder
Am 13. März 2008 organisierten die Stiftung Risiko-Dialog und die Schweizerische Akademie für Medizinische Wissenschaften SAMW im Zentrum Paul Klee in Bern eine Veranstaltung mit dem Titel «Rationierung im Gesundheitswesen». Da auch ich mittlerweile der Gruppe der offenbar besonders betroffenen Senioren angehöre, hat mich das Thema angezogen. Zweifellos war es ein sehr interessanter Nachmittag, hervorragend moderiert von Roger de Weck. Dennoch gestehe ich, dass ich etwas perplex heimgekehrt bin.

Ich dachte, dass sich besonders die Ärzte von der Thematik angesprochen fühlen würden, denn sie betrifft die Rationierung in erster Linie. Doch waren nur einige wenige unter den rund hundert Teilnehmenden vertreten - weder ein Vertreter der Fachgesellschaften noch der FMH -, und die meisten waren Grauköpfe wie ich.

Rund die Hälfte der Teilnehmer hielt eine Rationierung für nicht erforderlich, obgleich der Präsident der SAMW, Peter Suter, gute Argumente dafür vorbrachte. Er musste aber eingestehen, dass die Zahl von 2,7 Milliarden Franken ungerechtfertigter Ausgaben jährlich vermutlich zu niedrig angesetzt ist und es durchaus noch Luft für Rationalisierung anstelle von Rationierung gibt. Dennoch ist Rationierung nach Überzeugung von Nationalrätin Bea Heim Realität, und ältere Menschen fielen ihr als erste zum Opfer. Beispiel: Im Inselspital erhalten Senioren nicht dieselben kardiologischen Behandlungen wie jüngere Patienten. Aber Hand aufs Herz: Möchte ich, wenn ich einmal 85 bin, wirklich wie ein 30jähriger behandelt werden? Mein Tischnachbar, ein Pfleger in der Psychiatrie ein weiterer von der Rationierung bedrohter Bereich -, überzeugte mich, dass insbesondere Jugendliche von der Rationierung betroffen sein könnten.

Allgemein einig war man sich über die Definition der Rationierung: Sie liegt dann vor, wenn der Zugang zu den notwendigen Leistungen nicht mehr gewährleistet ist. Was als notwendig $\mathrm{zu}$ betrachten ist, ist allerdings nicht so einfach zu definieren. Und noch weniger, wer entscheiden soll. Der Bürger? In dieser Frage fand im Laufe des Nachmittags ein Meinungswandel statt: Nicht das Volk soll die Entscheidung treffen. Der Arzt (der tendenziell zuviel tut - so die Ansicht des santésuisse-Vertreters Stefan Kaufmann) oder der Patient? Oder beide gemeinsam?
Oder die Politiker? Letzteres schlug Peter Suter vor. Da winkte die Politikerin in der Runde sofort ab, da man die Antwort in diesem Fall erst in einem Jahrzehnt habe ...

Und schliesslich die Frage: Was ist notwendig und worauf könnte man verzichten? Auch in diesem Punkt gingen die Meinungen auseinander: Auf plastische Chirurgie oder Alternativmedizin? Auf eine Transplantation bei einem jungen Drogenabhängigen oder die Hüftprothese bei einem Neunzigjährigen?

Da glaubt man doch lieber, dass (noch) kein Bedarf an Rationierung besteht, obgleich diese bereits in grossem Umfang stattfindet. Doch auch die Rationalisierung ist kein einfaches Unterfangen. War der Angriff von seiten des BAG, vertreten durch Sandra Schneider, gegen die Psychiatrie wirklich gerechtfertigt, wo sie doch in der Zukunft zu unseren zentralen Gesundheitsfragen gehört? Sollen die Arzneimittelpreise gesenkt werden, obschon nachgewiesen ist, dass selbst ein teures Placebo besser wirkt als ein billiges? Oder sollen wir die banalen Erkrankungen aus dem Leistungskatalog streichen, wenngleich Erkältung und Rückenschmerzen die Gesellschaft aufgrund der von ihnen verursachten Arbeitsunfähigkeit teuer zu stehen kommen? Nein. Auch santésuisse muss lernen, dass die Medizin eine komplexe Disziplin ist.

Der Präsident von Risiko-Dialog, Matthias Haller, sprach von zwei «Gerechtigkeiten» in unserer Gesellschaft, einer Leistungs- und einer Bedarfsgerechtigkeit. Dem stimmten die anderen Redner $\mathrm{zu}$ - auch die Sozialdemokratin! Eine vollkommen gerechte Rationierung gibt es nicht. Zum Schluss war man sich ebenfalls einig, dass in der Schweiz auf hohem Niveau gejammert wird und weder die Politik noch eine nationale Kommission das Dilemma zwischen Rationalisierung und Rationierung lösen kann. So sei derzeit, fasste der Moderator zusammen, das muddling through die beste Lösung. Allgemeine Erleichterung: Denn «durchzuwursteln» entspricht schliesslich unserem Schweizer Naturell und häufig auch unserem Alltagsgeschäft als Ärzte - da, wo es keine EBM gibt. Und das ist der Fall für die Rationierung. Sie muss dort bekämpft werden, wo sie für grosse Ungerechtigkeit sorgt. Im Moment heisst die Devise Rationalisierung, und da gibt es noch Spielraum.
Hans Stalder*

\footnotetext{
und des Département de Médecine communautaire des Hôpitaux Universitaires de Genève.

Prof. Dr med. Hans Stalder, Facharzt für Innere Medizin FMH, Mitglied der Redaktion, war Leiter
} 